\title{
Situación actual de la disposición final de residuos sólidos en los municipios integrados en la Cuenca de la Laguna de Apoyo
}

\section{Ingrid Regidor*}

\section{Recibido: noviembre de 2010 / Aceptado: febrero de 2011}

El presente ensayo pretende proporcionar información acerca del estado actual de los vertederos municipales en los territorios integrados en la Cuenca de la Laguna de Apoyo, tomando en cuenta la reciente aprobación (mayo 2010) del Plan de Ordenamiento Territorial de la Cuenca de la Reserva Natural Laguna de Apoyo (RNLA). Además, procura servir a las alcaldías municipales como base para la planificación de proyectos para la mejora de los sitios de disposición final de los residuos sólidos.

Se abordan aspectos relacionados a la situación actual de la recolección de residuos sólidos municipales; los resultados sobre la disposición final de los residuos sólidos enmarcados en las características de la ubicación de los vertederos; el diseño, operación y mantenimiento de los mismos; y los aspectos administrativos y de control. Seguidamente se presenta una valoración de la ubicación, el diseño y la operación de los sitios de disposición final; la identificación y valoración de los impactos actuales y potenciales de carácter ambiental y social que pueden encontrarse en estos sitios. Por otro lado se plantean propuestas de mejora para el diseño y operación de los vertederos para mitigar los impactos potenciales identificados en ellos.

Palabras clave: disposición final de residuos sólidos / vertederos municipales / impactos ambientales / medidas ambientales 


\section{Introducción}

El deficiente manejo de los residuos sólidos en Nicaragua constituye una de las más serias amenazas para la salud de la población y para el medio ambiente. Algunos factores agravantes son: el acelerado crecimiento demográfico, el urbanismo, el desarrollo industrial, la producción a gran escala, los cambios en el estilo de consumo de la población a través de la difusión del estilo descartable, entre otros, ya sea por su concentración geográfica -muchas veces en áreas sobrecargadas o ambientalmente frágiles- o por la inserción de residuos progresivamente más peligrosos que conllevan a la contaminación del medio ambiente y al deterioro de los recursos naturales (Jaramillo, 1991).

A lo anterior se le suma la inadecuada aplicación de los servicios municipales de recolección, tratamiento y disposición final de residuos sólidos, además de la escasez de recursos económicos y la falta de personal capacitado en el manejo de estos residuos.

El efecto ambiental más obvio del manejo inadecuado de los residuos sólidos es el deterioro estético de las ciudades y el paisaje natural. Sin embargo existen otros efectos que contribuyen al agravamiento de este problema: la proliferación de vectores sanitarios, la contaminación de las aguas superficiales y subterráneas, la desvalorización tanto del terreno como de las áreas vecinas por el abandono y acumulación de los residuos sólidos a cielo abierto, la contaminación del suelo debido a las distintas sustancias que son depositadas en él, y los incendios y humos que reducen la visibilidad y pueden causar irritaciones nasales, oculares y afectaciones respiratorias.

Según el MARENA (2004), a nivel nacional se estima una generación total de residuos sólidos municipales de 3,500 Ton/día, equivalente a una producción anual de 1,277,500 Ton/año. Así mismo, para la producción per cápita se ha considerado una proyección global con una producción por habitante promedio de $0,45 \mathrm{~kg} /$ hab/día en el área urbana.

En cuanto a la disposición final, aproximadamente el 94\% de los residuos sólidos se dispone a cielo abierto y sin ningún tratamiento, causando graves problemas ambientales y de salud. No se aplican métodos de recuperación o reciclaje. Para la selección de los sitios a ser usados como vertederos municipales no se emplean los criterios y técnicas de evaluación de impacto ambiental y por otra parte, no hay control sobre estos sitios y solamente el 13\% de ellos cuenta con autorización sanitaria del MINSA (MARENA, 2004).

Esta problemática es parte de la realidad vivida en los municipios que forman parte de la cuenca de la Laguna de Apoyo -Catarina, San Juan de Oriente, Diriá, Diriomo, Niquinohomo, Granada y Masaya- que se encuentran constituidos en la Asociación de Municipios Integrados por la Cuenca y Territorios de la Laguna de Apoyo de Nicaragua (AMICTLAN). 


\section{Manejo de los residuos sólidos}

Según Castro y Regidor (2009), debido a la falta de recursos y capacitación técnica de los responsables de las municipalidades de Catarina, San Juan de Oriente, Diriá, Diriomo, Niquinohomo, Masaya y Granada en el tema de residuos sólidos y la falta de voluntad política por parte de las autoridades para llevar a cabo acciones acertadas en el manejo y disposición eficiente de los residuos sólidos, no se ha llevado a cabo, en algunos de ellos, un estudio que contemple el tipo y cantidad de residuos que se generan en las actividades cotidianas de los mismos.

Los residuos sólidos de San Juan de Oriente, Diriá y Diriomo no se encuentran caracterizados. No obstante, a manera general pueden ser clasificados según su composición en: materia orgánica, vidrio, metal y envases plásticos.

Para el caso de Niquinohomo, según el Proyecto de Mejoramiento de la gestión del servicio de limpieza pública municipal, para el año 2006 este municipio tenía una producción de residuos de $30 \mathrm{~m}^{3}$, de los cuales el 60\% era de naturaleza orgánica y el 40\% de carácter inorgánico (López, 2006).

Basándose en la caracterización de residuos realizada por INIFOM (2007) para el municipio de Catarina, los residuos sólidos generados en mayor cantidad son restos de comida $(51,41 \%)$, materia orgánica $(35,33 \%)$ y plástico $(5,32 \%)$. En el caso de Granada, los residuos sólidos de mayor generación son los restos de cocina (48\%), residuos de jardín (21\%), papel y plástico (8\% respectivamente), según el Proyecto para el mejoramiento del servicio de limpieza pública municipal de Granada, elaborado por la Alcaldía municipal en el año 2009 (Programa de Gobernabilidad y Desarrollo Local PROGEDEL - GTZ, Alcaldía Municipal de Granada \& Departamento de Servicios Municipales, 2009). Para Masaya la situación no varía mucho según lo expresado en el Plan maestro del manejo de residuos sólidos municipales (Alcaldía de Masaya, 2002). La composición de los residuos es muy parecida a lo anterior descrito: materia orgánica $(85,5 \%)$, papel y cartón $(4,8 \%)$, plástico $(3,2 \%)$ y tela $(2,5 \%)$.

A través de lo anterior expresado se constata que los residuos sólidos de los municipios mencionados son, en su mayoría, de composición orgánica biodegradable. Sin embargo, el volumen de residuos inorgánicos va en aumento.

De acuerdo a Lacayo Escobar (2009), la cobertura en relación con la prestación del servicio de recolección de residuos sólidos casa a casa a nivel nacional, es menor al 50\%, lo que se debe en gran medida a la ineficiencia en el servicio, a la escasa educación higiénico-sanitaria de la población, a la falta de recursos económicos de las municipalidades y al uso de tecnología inapropiada. Por otro lado, muchas veces los equipos y maquinarias con que se cuenta se hallan obsoletos, causando que las rutas realizadas no cubran las establecidas y los costos de recolección se incrementen por no poseer una planificación en las rutas (Lacayo Escobar, 2009).

A esta situación planteada no se escapan los municipios integrados en AMICTLAN. A continuación se muestra una breve descripción del escenario en que se desarrollan estas operaciones: 
Cuadro 1. Variables involucradas en la recolección de residuos sólidos en los municipios

\begin{tabular}{|c|c|c|c|c|c|c|c|c|}
\hline \multicolumn{2}{|c|}{ Variables involucradas en la recolección } & 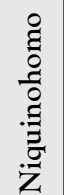 & 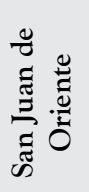 & 䋦 & 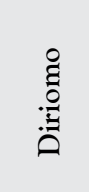 & ש̃ & 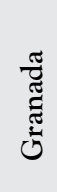 & 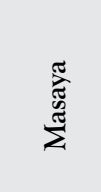 \\
\hline \multirow{2}{*}{$\begin{array}{l}\text { Cobertura del servicio } \\
\text { municipal (\%) }\end{array}$} & Urbano: & 70 & 100 & 100 & 100 & 85 & 84 & 93 \\
\hline & Rural: & 4 & 30 & $-*$ & 40 & - & - & - \\
\hline \multicolumn{2}{|c|}{ Cantidad de vehículos recolectores } & 2 & 1 & 1 & 2 & 1 & 4 & 6 \\
\hline \multicolumn{2}{|c|}{ Tipo de equipo recolector empleado } & $\frac{\stackrel{0}{0}}{\frac{\pi}{2}}$ & 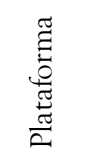 & 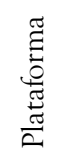 & 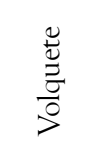 & 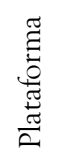 & 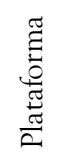 & 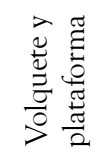 \\
\hline \multicolumn{2}{|c|}{ Capacidad de los vehículos recolectores $\left(\mathrm{m}^{3}\right)$} & 6 & 7 & 6 & 3 y 12 & 18 & 72 & 24 y 72 \\
\hline \multicolumn{2}{|c|}{ Número de trabajadores en la recolección } & 8 & 3 & 4 & 5 & 6 & 16 & 34 \\
\hline \multicolumn{2}{|c|}{ Frecuencia de recolección por semana } & 3 & 3 & 2 & 3 & 3 & 3 & 3 \\
\hline
\end{tabular}

* Para el municipio de Diriá y Catarina, el porcentaje de cobertura en la parte rural no se conoce, a pesar de cubrirse una pequeña parte de ésta.

Fuente: Castro y Regidor (2009)

Por otra parte, en estos municipios también se presentan botaderos ilegales, lo que dificulta a las alcaldías poder llevar a cabo un adecuado manejo y recolección de los residuos sólidos municipales. El número de botaderos varía de municipio a municipio y muchos de ellos se encuentran de forma permanente, siendo Diriomo -con seis botaderos- el que presenta mayor cantidad de botaderos y Niquinohomo -con dos botaderos- el de menor incidencia de este problema.

\section{Disposición final de los residuos sólidos}

En 2009 Lacayo Escobar expuso que existen muy pocas excepciones en los municipios del país en los que no se le brinda tratamiento final a los residuos sólidos, en la mayoría de ellos se disponen a cielo abierto con quemas ocasionales y proliferación de muchos vectores. La mayoría de estos vertederos han sido ubicados sin criterios técnicos, representando un serio riesgo para los ecosistemas y la salud de la población. Un problema que se suma a esta situación es la existencia de basureros no autorizados en los diferentes puntos del municipio que no cumplen con los principios y objetivos planteados. También se presenta como un obstáculo la carencia de los equipos necesarios para el acondicionamiento de los residuos en los vertederos y la falta de controles para el ingreso de recuperadores de materiales (pepenadores). 
En cuanto a los residuos sólidos considerados peligrosos, éstos no reciben el tratamiento adecuado, ya que son dispuestos sin ninguna separación en conjunto con los de naturaleza domiciliar. No se cuenta con un área específica donde se permita confinar este tipo de residuos, poniendo en riesgo a la población y al medio circundante.

La realidad vivida en los vertederos de los municipios integrados en AMICTLAN -según Castro y Regidor (2009) - no se aleja de lo anteriormente expuesto:

- Niquinohomo posee un vertedero municipal que tiene 15 años en operación, ocupado únicamente en un $4 \%$ del total del terreno, en el cual se dispone un área para recuperar residuos sólidos inorgánicos reciclables (plástico, vidrio, aluminio y chatarra). Posee una terraza de clasificación y acopio de material reciclable y una trinchera de compostaje en la que se utiliza tierra para cubrir aproximadamente $45 \mathrm{~m}^{3}$ de residuos sólidos que recibe diariamente. El camino de acceso a estas instalaciones no es asfaltado y posee una condición moderada.

Para la disposición de estos residuos no se utiliza control de vectores y a 20 $50 \mathrm{~m}$ de distancia se ubica la comunidad "Los Cocos". Por el momento no se cuenta con un plan de clausura. Además no tiene definida una capacidad de diseño.

- $\quad$ El vertedero de San Juan de Oriente recibe diariamente entre 7 y $14 \mathrm{~m}^{3}$ de residuos sólidos aproximadamente en un área que se ha ocupado en un 25\% y para los cuales no se aplica recubrimiento de tierra. En el terreno se lleva a cabo una separación de residuos (plástico) de manera informal. Se cuenta con una trinchera de residuos sólidos construida sin criterio técnico alguno y para la cual no se definió capacidad de diseño ni vida útil. Este vertedero cuenta con un único camino de acceso, no asfaltado y en condiciones moderadas, y se ubican fincas habitadas a $200 \mathrm{~m}$ aproximadamente.

- Diriá cuenta con un vertedero operado desde hace 12 años y ocupado en un $25 \%$, en el que se disponen aproximadamente $18 \mathrm{~m}^{3}$. La separación de los residuos sólidos se presenta de forma esporádica (plástico, vidrio aluminio y chatarra). No se posee ninguna capacidad de diseño, vida útil estimada, ni plan de clausura. El sitio no presenta infraestructura, ya que la disposición se realiza a cielo abierto y no hay control de vectores. El acceso al terreno es a través de un único camino no asfaltado y en condiciones difíciles. Se ubica a $500 \mathrm{~m}$ de distancia de la comunidad "La Zopilota".

- En Diriomo el vertedero municipal recibe desde hace 15 años $45 \mathrm{~m}^{3}$ de residuos sólidos aproximadamente, utilizándose hasta el momento el 25\% del terreno con una disposición que ocupa el área y rellena la depresión natural del terreno con material obtenido de una propiedad contigua, para la cual no se tiene control de vectores, por lo que tampoco se tiene definida una capacidad de diseño y un plan de clausura. Se dispone un área para separar residuos (plástico, vidrio, aluminio y papel) y se cuenta con un único camino de acceso, no asfaltado y en condiciones moderadas. 
- El vertedero municipal de Catarina se encuentra ocupado en un $26 \%$ recibiendo diariamente una cantidad aproximada de $23 \mathrm{~m}^{3}$ de residuos sólidos y realizándose una separación de los mismos de manera informal (aluminio, vidrio, plástico, hierro y cobre). Éste cuenta con dos trincheras construidas sin criterios técnicos, sin capacidad de diseño y a cielo abierto, por lo que se debe realizar una fumigación periódica. Lleva operando 15 años y se tiene prevista una vida útil de cinco años, sin considerarse por el momento un plan de clausura para el mismo. El terreno cuenta con un único camino de acceso, adoquinado y en buen estado, ubicándose a $1,000 \mathrm{~m}$ de un centro de salud y a $600 \mathrm{~m}$ de una urbanización.

- En Granada se depositan diariamente $240 \mathrm{~m}^{3}$ aproximadamente en un terreno que se encuentra ocupado en un $100 \%$, por lo que su vida útil ya está sobrepasada. Su capacidad de diseño estaba contemplada solamente para 20 años pero lleva operando 10 años más de lo previsto y a aún no se contempla un plan de clausura. Estos residuos son depositados a cielo abierto, por lo que se realiza fumigación periódicamente.

- De manera informal se da la separación de residuos (plástico, bronce, aluminio, hierro, cobre y sacos, entre otros). Posee un único camino de acceso, no asfaltado y en condición de difícil acceso y se ubica a $3000 \mathrm{~m}$ de la comunidad "El Pantanal", 1000 m de la comunidad "El Hormigón" y 2500 $m$ de la comunidad "La Fuente".

- En el caso de Masaya se cuenta con un vertedero que recibe aproximadamente $520 \mathrm{~m}^{3}$ de residuos sólidos a diario, los que han ocupado el 50\% del terreno, en el cual se realiza de manera informal una separación de residuos. Se cuenta con una trinchera construida sin criterios técnicos en la que se depositan los residuos y se compactan y cubren con tierra. Esta trinchera se diseñó para una capacidad de 20 años, de los cuales lleva funcionando nueve y se estima una vida útil de 15 años, para los que no se ha contemplado un plan de clausura. El vertedero cuenta con un único camino de acceso, no asfaltado y en condición moderada y se ubica a $500 \mathrm{~m}$ de un centro escolar de la comunidad "El Túnel".

Además de las condiciones antes mencionadas de los lugares ocupados como sitios de disposición final, es importante recalcar la situación de los mismos en cuanto a factores socioeconómicos y ambientales como:

- Distancia de fuentes de agua destinadas al abastecimiento:

Toda disposición final de residuos sólidos conlleva la generación de lixiviados ${ }^{1}$, sobre todo cuando ésta es en su mayoría de composición orgánica, como se describió anteriormente. Para los casos en estudio, únicamente el vertedero de Granada se encuentra ubicado aguas arriba de los pozos de agua potable que explota ENACAL 
para el consumo de la población, lo que representa una amenaza de contaminación al manto acuífero debido al inadecuado manejo de los residuos.

- $\quad$ Características del suelo:

Estos sitios presentan valores de profundidad desde los $40 \mathrm{~m}$ (Granada) hasta los 220 m (San Juan de Oriente, Diriá y Diriomo). La textura del suelo varía de arcillosa y franco-arcillosa hasta areno-arcillosa (Granada). La permeabilidad por lo general es moderada y la pendiente se presenta en valores de $0-15^{\circ}$ a $20,4^{\circ}$ (Catarina). Ninguno de los sitios presenta una pendiente mínima de $1 \%\left(0,5^{\circ}\right)$. Sin embargo la mayoría presenta valores bajos, a excepción de Catarina, por lo que esta condición puede ocasionar problemas de construcción de infraestructura en el terreno y drenaje de líquidos percolados.

- $\quad$ Condiciones de riesgo:

Los vertederos mencionados presentan la siguiente situación:

Cuadro 2. Condiciones de riesgo en los vertederos municipales

\begin{tabular}{|l|c|}
\hline Vertedero municipal & Tipo de riesgo presente \\
\hline Niquinohomo & Caída de tefra ${ }^{1}$ volcánica \\
\hline San Juan de Oriente & $\begin{array}{l}\text { Movimientos sísmicos por presencia de epicentros sísmicos reg- } \\
\text { istrados entre } 1975 \text { y } 2002^{2}\end{array}$ \\
\hline Diriomo & Ninguna \\
\hline Catarina & Ninguna \\
\hline Catarina & Derrumbes \\
\hline Granada & \\
\hline
\end{tabular}

Fuente: Castro y Regidor (2009)

\section{Diseño, operación y mantenimiento de los sitios de disposición final de los residuos sólidos municipales}

Los sitios de disposición final deberían presentar una infraestructura básica para garantizar tanto la seguridad del sitio como la de las personas que laboran en él. Sin embargo, según Castro y Regidor (2009) la siguiente situación es la que se muestra:

- $\quad$ En cuanto a la construcción de trincheras:

Los vertederos municipales de Niquinohomo, San Juan de Oriente, Catarina y Masaya, que son los que cuentan con trincheras, no se han construido bajo ningún criterio técnico y para San Juan de Oriente y Catarina a esto se le suma que depositan 
los residuos sólidos en ellas a cielo abierto.

Para el caso de Diriá no se cuenta con trinchera que permita depositar los residuos, sino que éstos son dispuestos sobre el nivel natural del suelo y no son cubiertos con material para evitar la formación de criadero de vectores.

Los residuos sólidos de Diriomo y Granada han ido llenando la depresión característica de los terrenos. En el caso del primero, antes de depositar los residuos generados se realizó una impermeabilización con geomembrana ${ }^{2}$ para evitar la infiltración de líquido lixiviado al suelo y con ello impedir también la contaminación del manto acuífero. Granada, en cambio, no cuenta con sistema o mecanismo alguno de impermeabilización o depósito de los residuos.

- $\quad$ En cuanto al drenaje perimetral de aguas pluviales:

Lamentablemente ninguno de los sitios de disposición final de los residuos de los municipios tomados en cuenta para esta valoración cuenta con un sistema de drenaje perimetral de agua pluvial. Por esta razón se supone que el agua de lluvia se combina con los residuos produciendo mayor volumen de lixiviados del que se generaría por acción de la descomposición de los residuos orgánicos.

- $\quad$ En cuanto al drenaje de lixiviado:

El vertedero del municipio de Niquinohomo cuenta con un pequeño drenaje de lixiviado ubicado en el interior de la zanja en donde son depositados los residuos de naturaleza orgánica. El lixiviado colectado por esta tubería es trasladado a una fosa donde es almacenado y tratado por acción de bacterias de nombre EM-1(abreviatura de Microorganismos Efectivos ${ }^{3}$ ) para luego descargarlo en un campo de irrigación. En el caso de los lodos, se reutilizarán como abono orgánico.

De igual manera, el vertedero del municipio de Diriomo cuenta con un sistema de drenaje para este tipo de líquidos. A diferencia del municipio anterior, el lixiviado colectado no es tratado de ninguna manera y sólo se almacena en una fosa.

En el caso de los municipios restantes, ninguno de ellos presenta un sistema de drenaje para lixiviados, lo que se debe al elevado costo que representa su instalación para el bajo presupuesto con el que cuentan estas alcaldías.

- En cuanto al drenaje de gases:

Los vertederos de los municipios de Granada, San Juan de Oriente, Diriá, Catarina y Masaya no cuentan con drenaje de gases producto de la descomposición de los residuos orgánicos. En éstos se ha presentado de forma esporádica auto combustión de los residuos, ocasionando numerosos incendios prolongados de hasta seis meses, como se dio en el vertedero de Masaya en el año 2002.

\footnotetext{
Lámina sintética fabricada a base de PVC, polietileno, caucho y otros compuestos que se utilizan para revestir rellenos sanitarios para evitar que sustancias contaminantes regresen al entorno a través de la lluvia y el viento.

3 Contienen bacterias fotosintéticas, bacterias de ácido láctico, levaduras y otros hongos, que al entrar en contacto con materia orgánica segregan vitaminas, minerales y antioxidantes que enriquecen el suelo y mejoran la microflora y el cultivo del suelo.
} 
Por el contrario, el sitio de disposición de Niquinohomo cuenta con un sistema de ventilación de tubo PVC que realiza una función similar a la de una chimenea, permitiendo el escape de los gases. Esta chimenea se encuentra ubicada de manera vertical desde el fondo de la trinchera hasta unos $30 \mathrm{~cm}$ por encima de la superficie. Ésta se encuentra ubicada solamente en uno de los extremos de la celda.

En el caso del municipio de Diriomo, también se encuentra presente un sistema de drenaje de gases que ha sido cubierto por la masa de residuos, por lo que probablemente no esté ejerciendo su función adecuadamente.

- $\quad$ En cuanto al cerco perimetral:

Seis de los siete sitios de disposición final poseen un portón en la entrada principal, exceptuando a Catarina -donde además el cerco perimetral se encuentra en muy mal estado. No obstante los cercos no cumplen con la función prevista debido a que se mantienen permanentemente abiertos, permitiendo el ingreso de personas y animales al terreno.

Los vertederos se encuentran rodeados de cercos de alambre de púas que se hallan deteriorados por la falta de mantenimiento, a excepción de Niquinohomo, que con el apoyo de organizaciones no gubernamentales ha logrado realizar mejoras en el sitio. De manera paralela al cerco se encuentra dispuesto un cerco vivo de árboles que ayuda a mitigar los posibles efectos negativos sobre los predios vecinos generados por el manejo de los residuos en el botadero.

- $\quad$ En cuanto a la caseta de control e instalaciones sanitarias:

Este tipo de infraestructura se encuentra en seis de los siete vertederos municipales visitados, a excepción de Granada. Desafortunadamente, sólo en el caso de Niquinohomo ésta se encontraba en buen estado, ya que en los cinco municipios restantes, tanto la caseta como las letrinas se encontraban en estado de abandono. Esta situación se debe a que solamente en los municipios de Masaya, Niquinohomo, Diriomo y Granada existe la presencia de un encargado del vertedero. En los demás municipios se encuentra ausente una persona que realice esta labor.

Por otro lado, tanto el botadero situado en Niquinohomo como el de Diriomo, presentan un área específica para realizar las actividades de separación, clasificación y almacenamiento de los residuos recibidos para luego venderlos a empresas recicladoras de este tipo de materiales.

- $\quad$ En cuanto al patio de maniobras:

A excepción del vertedero del municipio de Granada, todos cuentan con el espacio suficiente para que la unidad recolectora tenga la capacidad de maniobrar y depositar los residuos en el frente de trabajo.

En el vertedero de nombre "La Joya" (en Granada), además de no presentarse este espacio disponible, los residuos se encuentran dispuestos en una distancia de 1 $000 \mathrm{~m}$ antes del acceso principal al sitio debido a que en período de lluvia es muy difícil ingresar al terreno, por lo que las unidades recolectoras se ven obligadas a descargar a los lados del camino. 
- $\quad$ En cuanto al cartel de presentación:

Solamente las alcaldías municipales de Niquinohomo, Masaya y Diriomo han identificado sus sitios para la disposición final de los residuos. Ninguno de los sitos visitados posee un nombre específico. En todos los casos éstos son llamados "Vertedero Municipal" y en el caso particular de Masaya, el cartel tiene por nombre "Relleno sanitario", modo erróneo de nombrar al sitio ya que éste aún no cumple con las especificaciones técnicas para llamarse de esa manera.

- En cuanto al equipo y maquinaria de trabajo:

Debido a la falta de recursos económicos de las municipalidades, los vertederos cuentan con muy poca maquinaria y equipo de trabajo, lo que representa un impedimento para el eficiente manejo y disposición de los residuos en los mismos. Aunque poca, la maquinaria que poseen es propia, a excepción de Granada, que alquila una minicargadora.

Los municipios que tienen mayor deficiencia en este aspecto son Niquinohomo, Diriá, Catarina y San Juan de Oriente, ya que éstos solamente cuentan con las unidades de recolección y no poseen maquinaria para lo operación del vertedero.

Masaya y Granada tienen mayor acceso a equipo y maquinaria. En ambos sitios se cuenta con palas mecánicas utilizadas tanto para la distribución de los residuos en el área del terreno, como para tratar de compactarlos. Sin embargo, éstas no se dan a basto debido a las grandes cantidades de residuos que se reciben en estos dos municipios a causa de su posición como cabeceras departamentales.

El botadero de Diriomo, utiliza una carreta de bueyes a la que se le ha adicionado un rodillo grande que permite realizar el proceso de compactación de los residuos para disminuir el espacio que ocupan en el suelo.

El vertedero municipal de Masaya es el único que cuenta con una báscula que le permite al operador del mismo tener mayor conocimiento sobre la cantidad de residuos sólidos que se reciben diariamente.

\section{Aspectos administrativos y de control de la disposición final de los residuos sólidos}

Debido a que el manejo de los residuos sólidos y su disposición sanitaria final se encuentran determinados por la calidad de la administración local y la eficiencia de sus dirigentes municipales, es importante destacar ciertos aspectos relacionados al control y manejo de los vertederos municipales, ya que son éstos los que en gran medida determinan si existe voluntad política, capacidad de gestión y responsabilidad para brindar la debida protección de la salud pública y de los trabajadores, además de obtener un buen aspecto y protección del ambiente en el territorio municipal.

Por otra parte, el empleo de tecnología apropiada y de una buena planeación y administración, permite reducir los costos por la prestación del servicio y, por lo tanto, se logra una tarifa razonable que contribuya a su autofinanciamiento de acuerdo con la capacidad de pago del usuario de la localidad. 
Según Castro y Regidor (2009), en los vertederos municipales se presentan algunas condiciones incorrectas en cuanto a salud y seguridad, entre ellas se destacan:

- La falta de uso de ropa adecuada y equipos de protección personal.

- La ausencia de aseo personal al terminar la jornada de trabajo en los sitios en los que no se cuenta con el servicio básico de agua potable.

- La ingesta de alimentos en el frente de trabajo y la falta de lavado de manos con agua y jabón.

- $\quad$ Algunas veces se trabaja en jornadas excesivamente largas, con la consiguiente fatiga de los trabajadores.

- No se presta atención a la descarga del camión recolector de residuos, como en el caso de los vertederos de Masaya y Granada.

- $\quad$ Se permite el ingreso de personas ajenas al vertedero municipal, a excepción de Masaya, que cuenta con este tipo de control.

- Se queman los residuos sólidos en los vertederos de San Juan de Oriente, Niquinohomo y Catarina.

- No se les presta el debido mantenimiento a los equipos y herramientas de trabajo.

- No existe control en el ingreso de animales para consumo humano, circunstancia que pone en riesgo la salud de la población

Por otra parte, los vertederos municipales en estudio no poseen procedimientos o indicadores sobre el control en la disposición final de los residuos, situación que dificulta la evaluación de las condiciones de operación de los mismos. Las administraciones de estos sitios no cuentan con este tipo de información registrada de forma verificable, de tal forma que la mayoría de los datos que se manejan son inconsistentes y sin bases documentadas, por lo que no se puede comprobar si los datos proporcionados son fidedignos.

Las tarifas fijadas por el servicio de recolección y disposición final de los residuos municipales varían según las condiciones administrativas e institucionales, basadas en las políticas que establece cada municipio. Se puede decir que ninguna de las tarifas cobradas en estos municipios ha sido diseñada bajo criterios socioeconómicos, así como tampoco están siendo actualizadas según la devaluación de la moneda nacional, situación que limita el desarrollo de nueva infraestructura en la disposición final de los residuos. En Niquinohomo, Catarina, Granada y Masaya la tarifa fijada para la recolección es diferenciada según lo establecido en las Ordenanzas Municipales, y toma en cuenta los diferentes sectores de la localidad, desde las instituciones, sector comercial, industrias, mercados (para el caso de Masaya) hasta la fracción domiciliar. En el caso de San Juan de Oriente, Diriomo y Diriá, la tarifa de cobro por el servicio de recolección de los residuos sólidos municipales es fija, es decir que no se hace diferencia en los cobros entre las diferentes fuentes generadoras.

Con respecto a los datos relacionados a los ingresos y egresos generados en la prestación del servicio de recolección y disposición final de los residuos sólidos, los técnicos de las unidades ambientales en seis de los siete municipios no manejan este tipo de información, además de que en estas municipalidades no existe diferenciación 
entre las distintas operaciones de manejo de los residuos en los municipios (barrido de calles, recolección, transporte, tratamiento y disposición final). Únicamente Granada cuenta con matrices de la estructura de costos para cada servicio prestado y relación ingresos - egresos generados.

\section{Valoración de la ubicación, operación y mantenimiento actual de los sitios de disposición final de residuos sólidos municipales}

En 2009, Castro y Regidor evaluaron los vertederos de residuos sólidos municipales en cuanto a su localización, manera de operar y mantenimiento que reciben, tomando en cuenta una serie de consideraciones de tal forma que se prefiera aquellos lugares donde las operaciones sanitarias conduzcan a mejorar el terreno. Para valorar esta situación se tuvo que considerar los recursos técnicos y económicos disponibles en cada municipio.

Los criterios de selección del lugar presentan tres componentes importantes: económicos, ambientales y técnicos:

\section{-Factores económicos:}

- Costo y proceso de adquisición del terreno (tenencia)

\section{- Factores ambientales:}

- Distancia de fuentes de agua destinadas al abastecimiento

- Distancia horizontal hacia cualquier fuente de agua potable

- $\quad$ Profundidad del manto freático

- $\quad$ Coeficiente de permeabilidad bajo

- $\quad$ Protección a los recursos naturales

- Presencia de fallas geológicas

- Ubicación en zonas inestables (zonas de derrumbe, hundimientos naturales)

\section{-Factores técnicos:}

- $\quad$ Estado de las vías de acceso al sitio

- Tiempo de traslado desde el centro urbano

- Ubicación con respecto a los vientos

- Distancia al perímetro urbano

- Distancia a grupos de vivienda, industria, escuelas, hospitales, áreas de recreación

- Distancia de la vía principal

- Tipo de suelo

- $\quad$ Pendiente promedio mínima del terreno natural

- Compatibilidad con el desarrollo urbano

- Vida útil

- Cercanía de material de cobertura

- $\quad$ Propiedades del material de cobertura 
Tomando en cuenta los parámetros anteriores, Castro y Regidor (2009) evaluaron -utilizando como herramienta una matriz de valoración de sitios- cada vertedero municipal, obteniendo los siguientes valores para cada municipio:

Cuadro 3. Resumen de puntajes obtenidos en la valoración de sitios de los vertederos municipales

\begin{tabular}{|l|c|c|}
\hline \multicolumn{1}{|c|}{ Municipio evaluado } & Puntaje obtenido & Aptitud para relleno sanitario \\
\hline Niquinohomo & 56 & Buena \\
\hline Masaya & 54 & Buena \\
\hline Catarina & 47 & Mala \\
\hline Diriá & 46 & Mala \\
\hline Diriomo & 44 & Mala \\
\hline San Juan de Oriente & 43 & Mala \\
\hline Granada & 29 & Muy mala \\
\hline
\end{tabular}

Fuente: Castro y Regidor (2009)

De la misma forma en que se valoró el emplazamiento de los vertederos, se valoró la operación y mantenimiento de los mismos. Las condiciones que se tomaron en cuenta para llevar a cabo esta valoración fueron las siguientes:

- Área de acceso y espera dentro del relleno

- Cerco perimetral

- $\quad$ Caseta de control y vigilancia

- Camino de acceso buenas condiciones

- Área para futura expansión

- Drenajes perimetrales e internos

- Los drenajes perimetrales presentan un mantenimiento correcto

- Acceso a energía eléctrica el sitio

- Acceso a agua potable el sitio

- $\quad$ Presencia en el sitio de una zona de amortiguamiento

- $\quad$ Existencia de almacenes y bodegas para los equipos y herramientas de trabajo

- Área administrativa

- $\quad$ Estructura para el manejo de excretas

- Se cuenta con baños y vestidores para los operarios

- $\quad$ Posee el relleno la maquinaria y equipo suficiente de acuerdo a las características del mismo

Esta información fue empleada por Castro y Regidor (2009) para aplicar la matriz de valoración de la infraestructura, operación y mantenimiento de los vertederos municipales. Como se observa en el cuadro 4, todos presentan deficiencias, aunque unos más que otros. Estas deficiencias se deben a la falta de recursos económicos de las alcaldías, que impide el adecuado manejo de los residuos 
sólidos en los sitios de disposición final, acompañada de la falta de maquinaria y equipos necesarios para llevar a cabo las actividades de operación requeridas.

Cuadro 4. Puntajes de valoración del diseño y mantenimiento de obras complementarias que posee cada una de los vertederos municipales

\begin{tabular}{|l|c|c|}
\hline \multicolumn{1}{|c|}{ Municipio } & Puntaje de valoración & Criterios de valoración \\
\hline Niquinohomo & 23,0 & Aceptable \\
\hline Diriomo & 20,5 & Regular \\
\hline Masaya & 17,5 & Regular \\
\hline Diriá & 13,5 & Deficiente \\
\hline San Juan de Oriente & 13,0 & Deficiente \\
\hline Catarina & 5,5 & Deficiente \\
\hline Granada & 2,0 & Deficiente \\
\hline
\end{tabular}

Fuente: Castro y Regidor (2009)

\section{Impactos actuales y potenciales de carácter ambiental y social asociados a la disposición final de los residuos sólidos municipales}

Dado que el destino de los residuos sólidos de los municipios en estudio son vertederos municipales que no cumplen con las condiciones básicas para recibir un tratamiento adecuado, estos sitios son considerados como fuentes de contaminación y representan un riesgo para los diferentes componentes ambientales adyacentes a los mismos.

La inadecuada disposición de los residuos pone en peligro los cuerpos de agua superficiales y subterráneos, lo que, asociado a la quema frecuente de basura, puede tener implicaciones graves para la calidad de los suelos, el agua, el aire y las cadenas o redes alimentarias, y por ende, sobre la salud de la población. 
Cuadro 5. Factores ambientales involucrados en la valoración de los impactos ambientales y sociales en los vertederos municipales.

\begin{tabular}{|c|c|}
\hline Aspecto Ambiental & Factores involucrados \\
\hline Suelo & $\begin{array}{l}\text { - Área ocupada por residuos sólidos } \\
\text { - Presencia de residuo sólido domiciliar y/o industrial } \\
\text { - Incompatibilidad de uso de suelo } \\
\text { - Presencia de lixiviados }\end{array}$ \\
\hline Aire & $\begin{array}{l}\text { - Presencia de biogás } \\
\text { - Quema de residuos sólidos } \\
\text { - Presencia de olores desagradables }\end{array}$ \\
\hline Agua & - Presencia de lixiviados \\
\hline Flora & - Daños a la vegetación \\
\hline Fauna & $\begin{array}{l}\text { - Proliferación de fauna nociva } \\
\text { - Alteración de la fauna terrestre o acuática }\end{array}$ \\
\hline $\begin{array}{l}\text { Patrimonio } \\
\text { cultural }\end{array}$ & $\begin{array}{l}\text { - Cercanía a sitios de patrimonio histórico, religioso y turístico } \\
\text { - Cercanía a áreas de reserva o protección natural }\end{array}$ \\
\hline $\begin{array}{l}\text { Actividades } \\
\text { socioeconómicas }\end{array}$ & $\begin{array}{l}\text { - Presencia constante de grupos humanos } \\
\text { - Riesgos a la salud de los grupos humanos que viven en la } \\
\text { zona o en los alrededores } \\
\text { - Riesgos de contaminación de animales de consumo humano } \\
\text { - Afectación de otras actividades (socioeconómicas, turísticas, } \\
\text { etc.) }\end{array}$ \\
\hline
\end{tabular}

Fuente: Castro y Regidor (2009)

Considerando estas condiciones, Castro y Regidor (2009) evaluaron cada vertedero municipal con el fin de conocer la magnitud de su impacto al medio ambiente. A continuación se presentan los resultados de la valoración de los impactos causados por la disposición final de los residuos sólidos:

Cuadro 6. Puntajes obtenidos en la evaluación de riesgo ambiental en los vertederos municipales

\begin{tabular}{|l|c|c|}
\hline \multicolumn{1}{|c|}{ Municipio } & Puntaje & Perturbación \\
\hline Granada & 27 & Fuerte \\
\hline Catarina & 24 & Medio \\
\hline Diriá & 23 & Medio \\
\hline Masaya & 23 & Medio \\
\hline Diriomo & 21 & Medio \\
\hline San Juan de Oriente & 21 & Medio \\
\hline Niquinohomo & 11 & Bajo \\
\hline
\end{tabular}

Fuente: Castro y Regidor (2009) 
El análisis de estos resultados revela que el vertedero de Granada es el sitio con mayor impacto ambiental, siendo el que obtuvo mayor puntuación, lo que significa que posee un grado de perturbación fuerte. En cambio, Catarina, Masaya, Diriá, San Juan de Oriente y Diriomo presentan una perturbación ambiental media. Por último, Niquinohomo presenta un bajo impacto ambiental.

A pesar de que el vertedero de Granada no se encuentra ubicado cerca de aguas superficiales, el manto freático de este terreno es muy superficial, lo que puede provocar que se presente contaminación de las aguas subterráneas, generada a partir de formación de los lixiviados. Debido a esta situación, el manto acuífero de Granada se encuentran en riesgo de verse afectado por los lixiviados provenientes de la disposición final de los residuos sólidos.

Con respecto a la contaminación del aire, todos los vertederos se ven afectados. Dado que la composición de los residuos es en su mayoría biodegradable, éstos son convertidos en biogás, lo que genera una afectación de ámbito local con olores, ruidos, incendios y explosiones, con efectos negativos para la salud de la población aledaña.

Los únicos vertederos que contrarrestan esta situación son Niquinohomo y Diriomo por poseer dentro de sus infraestructuras -aunque precario- un drenaje de gases colocado en la trinchera de residuos.

Los incendios y explosiones son otros de los impactos provocados por una inadecuada gestión del biogás. Los procesos de descomposición anaeróbica producidos en los vertederos generan metano, responsable de explosiones y combustiones espontáneas debido a su elevada inflamabilidad y a la capacidad para formar mezclas explosivas con el aire (Garrido, Márquez \& Garrido, 2008). Esta situación se presenta en seis de los vertederos y con mayor severidad en el de Masaya en donde para el año 2008 se presentó un incendio de gran magnitud que tuvo una duración de aproximadamente seis meses.

Finalmente se presentan otros ejes de contaminación atmosférica en el ámbito local de los vertederos: la generación de polvo o el transporte de materiales livianos, la afectación al paisaje, el ruido y vibraciones que son consecuencia del tráfico, la descarga de los camiones recolectores y el movimiento de los equipos de compactación (Calvo, 2003, citado por Garrido, Márquez \& Garrido, 2008).

Los impactos de los vertederos sobre el suelo pueden concretarse en su destrucción directa mediante arrastre o compactación dependiendo de la magnitud del impacto de la superficie destruida y de la calidad edáfica de la superficie ocupada (Hernández et al., 1998, citado por Garrido, Márquez \& Garrido, 2008). Ninguno de los sitios de disposición final estudiados escapa a este tipo de afectación debido a que los suelos de estos terrenos no se encuentran impermeabilizados, a excepción de Diriomo.

Con respecto a las afectaciones a la salud en estos sitios se ha presentado proliferación de vectores debido a la falta de control en el manejo de la disposición final. Esta situación se ha hecho notoria -según Castro y Regidor (2009)- a través de las quejas presentadas por parte de la población que habita en las áreas aledañas y se ha tratado de remediar a través de fumigaciones.

El manejo inadecuado y la deficiente disposición de residuos sólidos de estos vertederos municipales afectan significativamente el paisaje, deteriorándolo al recibir 
los residuos de todas las actividades del municipio. Por lo tanto, su inadecuado manejo no sólo afecta la salud y el ambiente, sino que disminuye la calidad de vida en términos del espacio y del horizonte.

\section{Algunas medidas ambientales de solución a los impactos ambientales}

A continuación se detallan, a manera general, algunas de las medidas ambientales que pueden implementarse en los sitios de disposición final.

Tabla 7. Medidas ambientales a ser implementadas para mitigar algunos de los impactos ambientales presentados en los vertederos municipales.

\begin{tabular}{|c|c|}
\hline $\begin{array}{l}\text { Condición } \\
\text { impactante }\end{array}$ & Medida ambiental \\
\hline $\begin{array}{l}\text { Generación de } \\
\text { aguas residuales } \\
\text { y lixiviados }\end{array}$ & 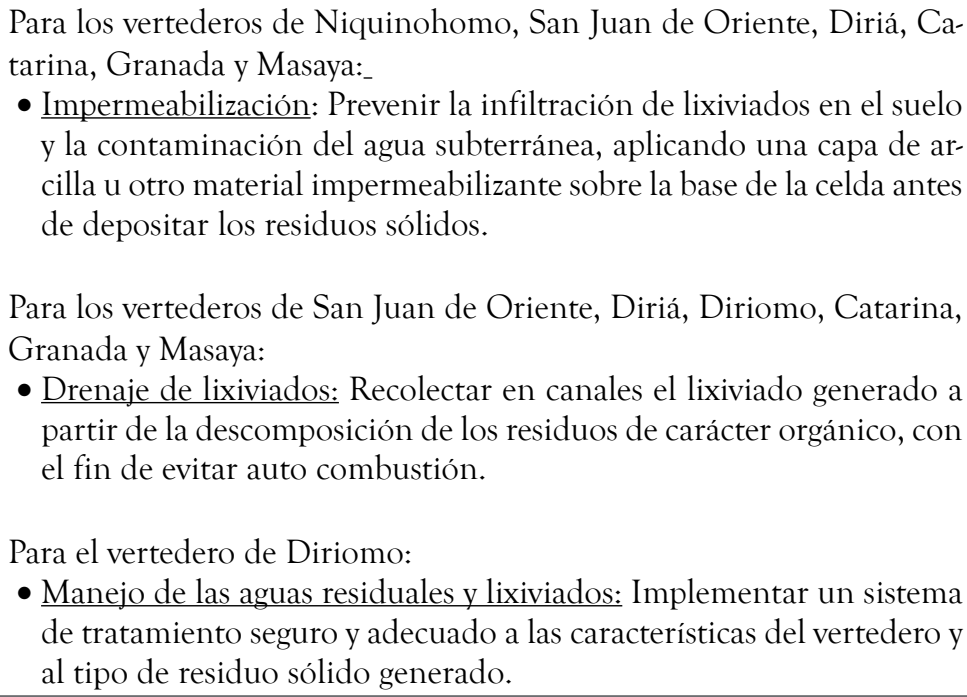 \\
\hline $\begin{array}{l}\text { Presencia de } \\
\text { aguas pluviales }\end{array}$ & $\begin{array}{l}\text { Para Niquinohomo, San Juan de Oriente Diriá, Diriomo, Catarina, } \\
\text { Granada y Masaya: } \\
\text { - Manejo del drenaje de aguas pluviales en las trincheras: Recolectar } \\
\text { el agua pluvial en canales para ser desviada y evitar que se mezcle } \\
\text { con los residuos. }\end{array}$ \\
\hline $\begin{array}{l}\text { Emisiones de } \\
\text { gases }\end{array}$ & $\begin{array}{l}\text { Para todos los vertederos: } \\
\text { - Tubería de ventilación: Colocar tuberías en diferentes puntos de la } \\
\text { celda de disposición de residuos para proporcionar una ruta en la } \\
\text { que el gas de la trinchera salga hacia la atmósfera. } \\
\text { - Quema de gases: Oxidar los gases volátiles y olorosos a compuestos } \\
\text { no olorosos a través de la implementación de quemadores de gases } \\
\text { en las chimeneas (conversión de } \mathrm{CH}_{4} \text { a } \mathrm{CO}_{2} \text {, de mayor a menor } \\
\text { contaminante). }\end{array}$ \\
\hline
\end{tabular}




\begin{tabular}{|c|c|}
\hline $\begin{array}{l}\text { Condición } \\
\text { impactante }\end{array}$ & Medida ambiental \\
\hline $\begin{array}{l}\text { Riesgo de con- } \\
\text { taminación y } \\
\text { accidentes del } \\
\text { personal por } \\
\text { falta de medidas } \\
\text { de seguridad y } \\
\text { protección. }\end{array}$ & $\begin{array}{l}\text { Para todos los vertederos (excepto Masaya): } \\
\text { - Control del acceso: Regular el ingreso de personas y animales al vert- } \\
\text { edero. } \\
\text { - Uso de EPP*: Garantizar la seguridad a la persona que labora en el } \\
\text { vertedero (guantes, mascarillas, botas y uniforme). } \\
\text { Para los vertederos de Catarina y Granada: } \\
\text { - Equipamiento del vertedero: Colocar un portón y cerco perimetral } \\
\text { en buen estado que permita mantener un acceso restringido de per- } \\
\text { sonas externas a la alcaldía municipal. Mejorar las condiciones de la } \\
\text { caseta de control con el fin de proporcionar el medio adecuado para } \\
\text { los trabajadores del lugar. }\end{array}$ \\
\hline $\begin{array}{l}\text { Malos olores y } \\
\text { proliferación de } \\
\text { vectores }\end{array}$ & $\begin{array}{l}\text { Para San Juan de Oriente, Diriomo, Catarina, Granada y Masaya: } \\
\text { - Compactación y cobertura: Reducir el volumen de residuos sólidos } \\
\text { mediante la compactación y disminuir la generación de vectores, } \\
\text { malos olores y esparcimiento de los residuos sólidos en el terreno, } \\
\text { aplicando un } 20 \% \text { de material de cobertura (suelo de la zona) del } \\
\text { total de los residuos sólidos depositados dentro de la celda. }\end{array}$ \\
\hline $\begin{array}{l}\text { Esparcido, } \\
\text { compactación y } \\
\text { cobertura de los } \\
\text { residuos sólidos }\end{array}$ & 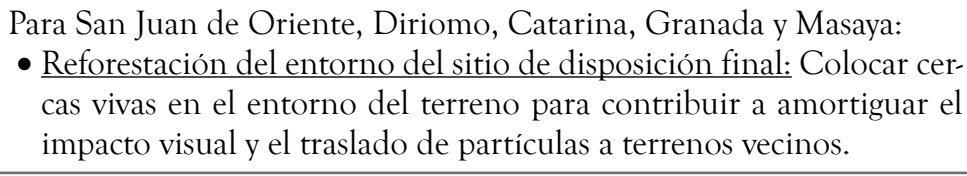 \\
\hline
\end{tabular}

* EPP: Equipo de protección personal

Fuente: Castro y Regidor (2009)

\section{Espacios requeridos en los vertederos para seguir almacenando residuos sólidos}

Con el fin de conocer los espacios que requieren los sitios de disposición final para recibir los residuos sólidos de la población municipal en los próximos 20 años, Castro y Regidor (2009), llevaron a cabo proyecciones de espacio.

La proyección se llevó a cabo para todos los municipios estudiados. Sin embargo, los vertederos de Masaya y Niquinohomo son los únicos que tienen posibilidades de continuar operando en función de un relleno sanitario en el terreno que poseen en la actualidad, debido a que la extensión de los mismos y sus características lo permiten. Los demás sitios deben ser clausurados y reubicados debido a las prácticas inadecuadas de operación, y por la vulnerabilidad social y ambiental del área en donde se encuentran ubicados.

Los resultados de la proyección -realizada en base al crecimiento de la población (proyectada a partir del año 2009)- que determina el área necesaria para 
continuar operando en los sitios actuales con la realización de las adecuadas técnicas de operación se muestran a continuación:

Cuadro 8. Comparación del área de terreno actual para la disposición final y el área requerida en 20 años para la operación de los vertederos municipales actuales con diseño y mantenimiento de rellenos sanitarios.

\begin{tabular}{|l|c|c|c|}
\hline \multirow{2}{*}{\multicolumn{1}{|c|}{ Municipio }} & Área actual & \multicolumn{2}{c|}{ Área requerida } \\
\cline { 2 - 4 } & Área $(\mathbf{m z})$ & Área $(\mathbf{m z})$ & Área $(\mathrm{Ha})$ \\
\hline Niquinohomo & 2,000 & 5,900 & 4,000 \\
\hline Masaya & 25,00 & 50,78 & 36,00 \\
\hline San Juan de Oriente & 1,998 & 2,070 & 1,000 \\
\hline Diriomo & 3,995 & 8,420 & 6,000 \\
\hline Diriá & 1,998 & 2,200 & 2,000 \\
\hline Catarina & 2,695 & 2,800 & 2,000 \\
\hline Granada & 2,995 & 45,45 & 32,00 \\
\hline
\end{tabular}

Fuente: Castro y Regidor (2009)

Como se observa, el área que abarcan actualmente los vertederos municipales con posibilidades de extensión (Niquinohomo y Masaya) es de menor tamaño a la que requieren para seguir operando en los próximos 20 años, por lo que, aunque estos sitios tienen la oportunidad de ser diseñados y operados como relleno sanitario, el tiempo de vida útil que resta para cada uno de ellos es de ocho años como máximo.

El resto de sitios de disposición final de residuos sólidos deben implementar medidas que disminuyan el riesgo ambiental y social de los municipios durante el período de trabajo faltante. Una vez alcanzada la capacidad de carga de estos vertederos, se debe realizar un análisis de alternativas para la reubicación de dichos sitios en base a los datos de extensión del área requerida para cada uno de ellos y considerando los parámetros establecidos en la NTON 05013 - 01 (MARENA, 2001).

Cuadro 9. Área de terreno requerida en ocho años para la operación de los vertederos municipales actuales con diseño y mantenimiento de rellenos sanitarios

\begin{tabular}{|l|c|c|}
\hline Municipio & Área $(\mathrm{mz})$ & Área $(\mathrm{Ha})$ \\
\hline Niquinohomo & 1,59 & 1,00 \\
\hline Masaya & 14,49 & 10,00 \\
\hline
\end{tabular}

Por esta razón, antes de cumplir este plazo mínimo de operación se deben seleccionar nuevos sitios que cumplan con los parámetros establecidos en la NTON 05013 - 01 (MARENA, 2001). 


\section{Conclusiones}

El manejo de los residuos sólidos en cada uno de los municipios integrados en la Cuenca de la Laguna de Apoyo es sub valorado por parte de las autoridades municipales. Las alcaldías prestan un deficiente servicio de recolección a la población; el porcentaje de cobertura de este servicio en la mayoría de los municipios no abarca el $100 \%$, poniendo en riesgo la salud de la población y obligando a las personas a buscar alternativas inadecuadas (como la quema o la creación de botaderos ilegales) para la disposición final de los residuos.

- La dirección de servicios municipales de cada alcaldía carece de información documentada acerca de la recolección y disposición final de los residuos, lo que provoca que:

- No se tenga establecido un mapa de macro y micro ruteo debido a la falta de capacidad en el diseño de rutas, lo que conduce a un desorden al momento de llevar a cabo la operación y a un incremento en los costos por gastos en combustible y aumento de los tiempos de recolección.

- No se cuente con información relacionada a la caracterización de los residuos sólidos, volumen y cantidad que se genera por cada fuente en los municipios de San Juan de Oriente, Diriá y Diriomo, y en el caso de Niquinohomo, Catarina, Granada y Masaya los datos estén desactualizados.

- $\quad$ No se maneje de forma documentada la producción per cápita de residuos sólidos municipales y el volumen que ingresa a los vertederos para ser dispuestos finalmente (a excepción del vertedero de Masaya).

- No se conozca los costos de operación y los ingresos recibidos por la prestación del servicio, por lo tanto no sea posible determinar su auto-sostenibilidad.

- No se conozca a ciencia cierta la capacidad de diseño con que en un inicio estos vertederos fueron construidos y tampoco se cuente con planes de clausura y post-clausura para los mismos.

Esta situación se ve agravada con cada cambio de administración municipal, en el que se pierde la secuencia de las acciones realizadas y se empieza desde cero en las actividades propuestas, dejando en el olvido y sin respaldo las metas alcanzadas en el manejo integral de los residuos sólidos. Además, existe una deficiencia de maquinaria y equipos de operación, ligada directamente con la falta de recursos económicos de las alcaldías municipales.

El servicio de recolección de estos municipios no cuenta con una tarifa regulada y el valor cobrado mínimo; existen serias deficiencias en el cobro, ya sea por debilidades administrativas o por problemas relacionados a la educación comunitaria. Esta circunstancia se presenta a pesar de existir en algunos de los municipios -Niquinohomo y Masaya- una ordenanza municipal donde se detalla los cobros por los servicios prestados. En otros casos ni siquiera se tiene establecido un cobro diferenciado para cada tipo de fuente. Esta situación acrecienta la problemática existente alrededor de la desintegración de los ingresos a dichos servicios provocando 
su debilitamiento.

Actualmente, en estos sitios se desarrolla de manera informal la separación y clasificación de los residuos sólidos con características reciclables, poniendo en riesgo la vida de las personas que desempeñan esta función dentro de los vertederos, ya que no se utiliza ningún mecanismo de control y vigilancia para desarrollar esta actividad y en todos, a excepción de Niquinohomo, es llevada a cabo por personas externas a las alcaldías.

Por otra parte, la falta de un manejo integral de los residuos sólidos en estos municipios provoca que:

Todos los territorios presenten una limitante para catalogarse como área idónea para convertirse en rellenos sanitarios, no obstante los sitios con mayores posibilidades se encuentran en los municipios de Niquinohomo y Masaya.

Una mala disposición y manejo de los residuos en los vertederos municipales, lo que ha ocasionado que éstos hayan reducido su vida útil en menor tiempo del estipulado, y que incluso ya no tengan capacidad de recibir más residuos, como sucede con el municipio de Granada.

Ninguno de los vertederos municipales cuente con las instalaciones y estructuras necesarias para llevar a cabo una adecuada y segura disposición final de los residuos sólidos, ya que tanto los que construyeron trincheras como los que emplearon el método del área para disponer los residuos, no emplearon criterios técnicos para su diseño. En cuanto a la evaluación del diseño y operación en general, sólo los vertederos de Niquinohomo y Masaya poseen condiciones que se acercan a una infraestructura y equipamiento idóneo, y realizan las prácticas más adecuadas para el manejo de los residuos sólidos en el sitio.

$\checkmark \quad$ No se utilicen técnicas o mecanismos que garanticen la seguridad y bienestar laboral de los trabajadores en estos sitios, lo que representa un factor de riesgo para la salud de los mismos.

Falte previsión ante los posibles problemas que pudieran presentarse, ya sean de carácter técnico, administrativo o de control, lo que ocasiona que la disposición final de los residuos sólidos sea vista como un problema de gran impacto ambiental, que puede tener afectaciones a nivel local o regional.

Aparezcan impactos ambientales que pueden traer graves repercusiones para los municipios en estudio, y en muchos de los casos éstos puedan ser irreversibles, como en Granada con el latente riesgo de la contaminación de los acuíferos. 


\section{Recomendaciones}

Para resolver las condiciones limitantes presentadas alrededor del manejo y disposición final de los residuos sólidos se detallan a continuación algunas recomendaciones:

$\checkmark \quad$ Es necesario que las municipalidades cuenten con un programa integral de manejo de residuos sólidos que incluya:

- $\quad$ El diseño de las rutas de macro y micro ruteo, lo que contribuirá a tener una mayor eficiencia en la recolección a través de la optimización de las distancias y los tiempos de operación.

- El mantenimiento preventivo a las unidades de recolección con las que cuentan las municipalidades para evitar su deterioro y eliminar las probabilidades de atrasos en el proceso debido a la falta de equipos en buen estado.

- La definición de una política relativa al manejo adecuado, integral y ambiental de los residuos, apropiada a la naturaleza, escala e impactos ambientales potenciales de las actividades, productos y servicios que comprende dicho manejo en cada caso particular. Esto se podría lograr a través de la elaboración de planes sectoriales de manejo de residuos sólidos ajustados a la realidad de cada municipio, con el fin de encontrar posibles soluciones a los problemas evidentes.

- La elaboración y fortalecimiento de programas de divulgación y educación ambiental desde las alcaldías para fomentar en la población el reciclaje y la reutilización, de tal forma que ello contribuya a disminuir la generación de residuos.

- La elaboración de tarifas sólidas que cubran el costo total del servicio de recolección y disposición final, aspecto crucial para lograr su autofinanciamiento y mejorar la calidad del servicio.

- Identificar y documentar los aspectos ambientales (emisiones al aire, aguas residuales, generación de residuos, fugas y derrames, etc.) involucrados en las distintas áreas o etapas del sistema de manejo integral de los residuos sólidos, de tal forma que con ello se puedan establecer medidas para su prevención, control y mitigación ante cualquier afectación de este tipo.

- Diseñar una celda de confinamiento en los vertederos municipales -debido a que la mayoría de las instituciones de salud (hospitales, clínicas, centros de salud, etc.) de estos municipios no cumplen con las condiciones de operación de la disposición final (incineradores) de los residuos sólidos biológicos infecciosos- con el fin de evitar proliferación de vectores y contagio a las personas en contacto directo con éstos.

- $\quad$ Conociéndose que los vertederos de San Juan de Oriente, Diriá, Diriomo y Catarina no prestan las condiciones necesarias en cuanto a diseño, operación y mantenimiento, para seguir disponiéndose en ellos los residuos sólidos, es necesario que sean rehabilitados lo más pronto posible con el fin de lograr una disposición final de residuos de forma segura y adecuada. De esta manera, 
estos lugares se podrían seguir utilizando sin ningún tipo de riesgo, aplicando medidas ambientales para que éstos cumplan con las especificaciones de la NTON 05 013-01 (MARENA, 2001).

- $\quad$ Para el caso del vertedero de Granada, éste debe ser clausurado urgentemente debido a que la mala disposición de los residuos sólidos y la falta de condiciones para que éstos sean tratados, provoca un fuerte impacto ambiental. Por lo tanto, es necesario que se realice un análisis de alternativas de sitios con el fin de tomar decisiones fundamentadas en la disposición final de los residuos y afines al cumplimiento de las especificaciones de la NTON 05 013-01 (MARENA, 2001).

- $\quad$ Es importante realizar un reordenamiento de los sitios de disposición final de residuos sólidos de tal forma que se pueda contemplar un área específica destinada para la separación y clasificación de los residuos, en la que pueda llevarse a cabo esta actividad sin ningún tipo de peligro para las personas encargadas de la misma.

- A largo plazo es importante que los municipios, dentro de sus planes municipales de gestión integral de residuos, tengan en cuenta el reciclaje a partir de la separación en la fuente y la recolección selectiva, es decir, que los generadores de residuos (viviendas, comercio, industria) los presenten en forma separada para que sobre ellos se pueda realizar una gestión diferencial adecuada.

\section{Referencias bibliográficas}

Alcaldía Municipal de Masaya. Departamento de Salud y Limpieza. (2002). Plan Maestro. Manejo de desechos sólidos. Manuscrito no publicado.

Castro M. \& Regidor, I. (2009). Valoración socio-ambiental de los sitios de disposición final de residuos sólidos de los municipios integrados en la Asociación de Municipios Integrados por la Cuenca y Territorios de la Laguna de Apoyo de Nicaragua (AMICTLAN). Estudio monográfico para optar al Título de Ingenieras en Calidad Ambiental, Universidad Centroamericana, Managua, Nicaragua.

Garrido, M., Márquez, F. \& Garrido, P. (2008). Accidentes provocados por los vertederos de residuos sólidos urbanos. Recuperado el 18 de noviembre de 2009 de http:// www.serviprevencia.org/IMG/pdf/accidentes_en_vertederos_de_residuos. pdf.

Instituto Nicaragüense de Fomento Municipal INIFOM - PRODELFIS/GTZ. (2007). Caracterización de los residuos sólidos utilizando el método del cuarteo. Municipio de Catarina. Manuscrito no publicado.

Jaramillo, J. (1991). Guia para el diseño, construcción y operación de rellenos sanitarios manuales. Serie Técnica No. 28. Washington, D.C: Organización Mundial de la Salud y Organización Panamericana de la Salud.

López, D. (2006). Proyecto de mejoramiento de la gestión del servicio de limpieza pública municipal de Niquinohomo. Manuscrito no publicado. 
MARENA. (2004). Bases de la Politica Nacional sobre la Gestión Integral de los Residuos Sólidos. Managua: MARENA.

MARENA (2001). NTON 05013 - 01. Norma técnica para el control ambiental de los rellenos sanitarios para desechos sólidos no peligrosos. Recuperado el 20 de noviembre de 2009 de http://www.marena.gob.ni/index.php?option=com remository\& Itemid=181\& func $=$ showdown $\&$ id $=232 \&$ date $=2010-02-01$.

Lacayo Escobar, M. (2009). Manejo integral de los residuos sólidos urbanos. Managua:

Manuscrito no publicado.

Programa de Gobernabilidad y Desarrollo Local PROGEDEL - GTZ. Alcaldía Municipal de Granada. Departamento de Servicios Municipales (2009). Proyecto de Mejoramiento del servicio de limpieza pública municipal de Granada. Manuscrito no publicado.

\section{Anexo}

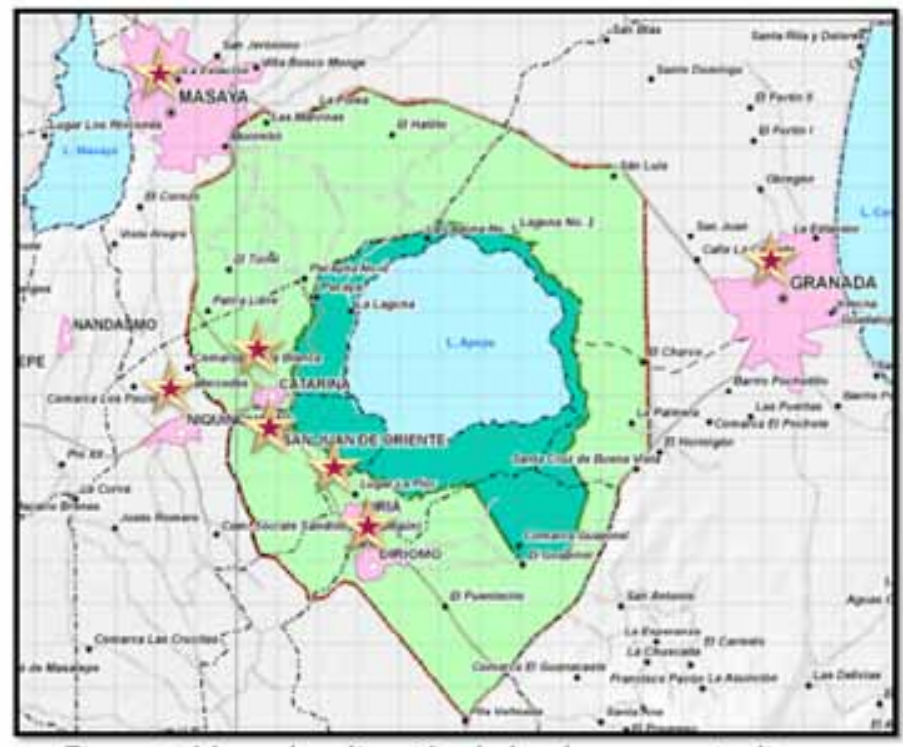

Universos

Municipios de:

- Masaya

- Catarina

- Granada

- San Juan de Oriente

- Diriá

- Diriomo

- Niquinohomo

Figura 1. Macro localización de las áreas en estudio

Ilustración 1. Ubicación de los vertederos municipales integrados en los territorios de la Cuenca de la Laguna de Apoyo

Fuente: Castro y Regidor (2009), tomado a partir de Mapa de Ubicación de la RNLA en su contexto regional, AMICTLAN (2009). 